\title{
Improving the Documentation of Endotracheal Intubation in a Tertiary Neonatal Unit
}

\author{
K Jamieson, S Hubbard and S Jaques - Evelina Children's Hospital, London
}

\section{Background}

Safe airway management in a neonatal intensive care setting is crucial to optimise patient care and prevent mortality and morbidity. Many factors contribute to difficulties in intubation such as patient factors, technical issues and human factors. This provides an opportunity for development of standard operating procedures and documentation. We aimed to evaluate the effect of a checklist on the adequacy of documentation of intubation in a neonatal intensive care setting.

\section{Methods}

Data collection was retrospective, pre and post implementation of a bespoke intubation checklist/sticker. All babies undergoing intubation by the neonatal team in a level 3 neonatal unit, during randomly selected pre- and postimplementation 3 week periods were identified. Set criteria for 'complete documentation' of intubations were defined.

Criteria for complete documentation:

- Tube size

- Tube length at lips

- Number of attempts required

- Reason for intubation

- Person performing intubation

- Tube position confirmed with $\mathrm{CO}_{2}$ colour change

- X-ray performed and position confirmed

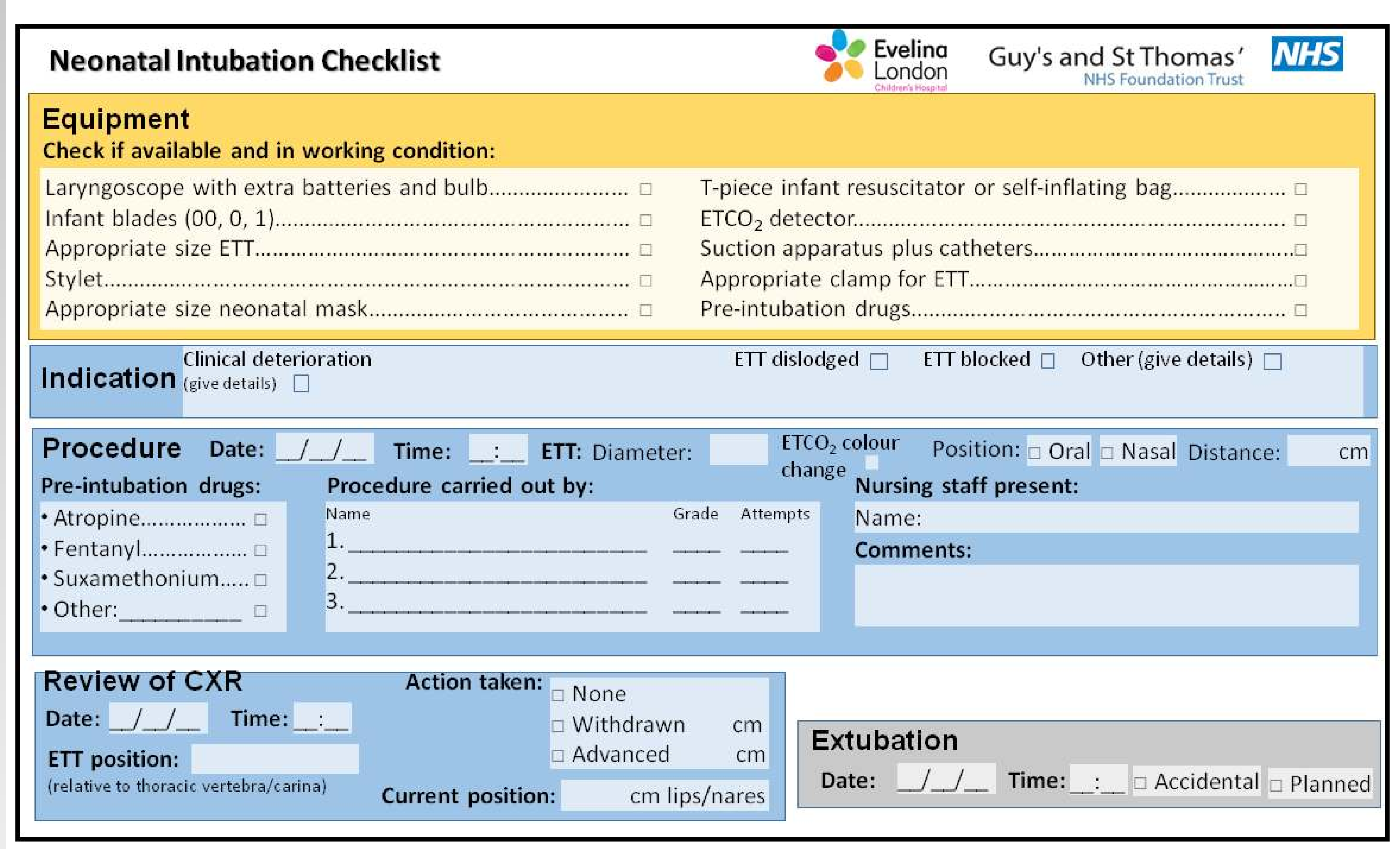

\section{Results}

Pre-implementation period: 20 intubations Post-implementation period: 28 intubations Sticker used for 23 of 28 post-implementation intubation events

Post-implementation

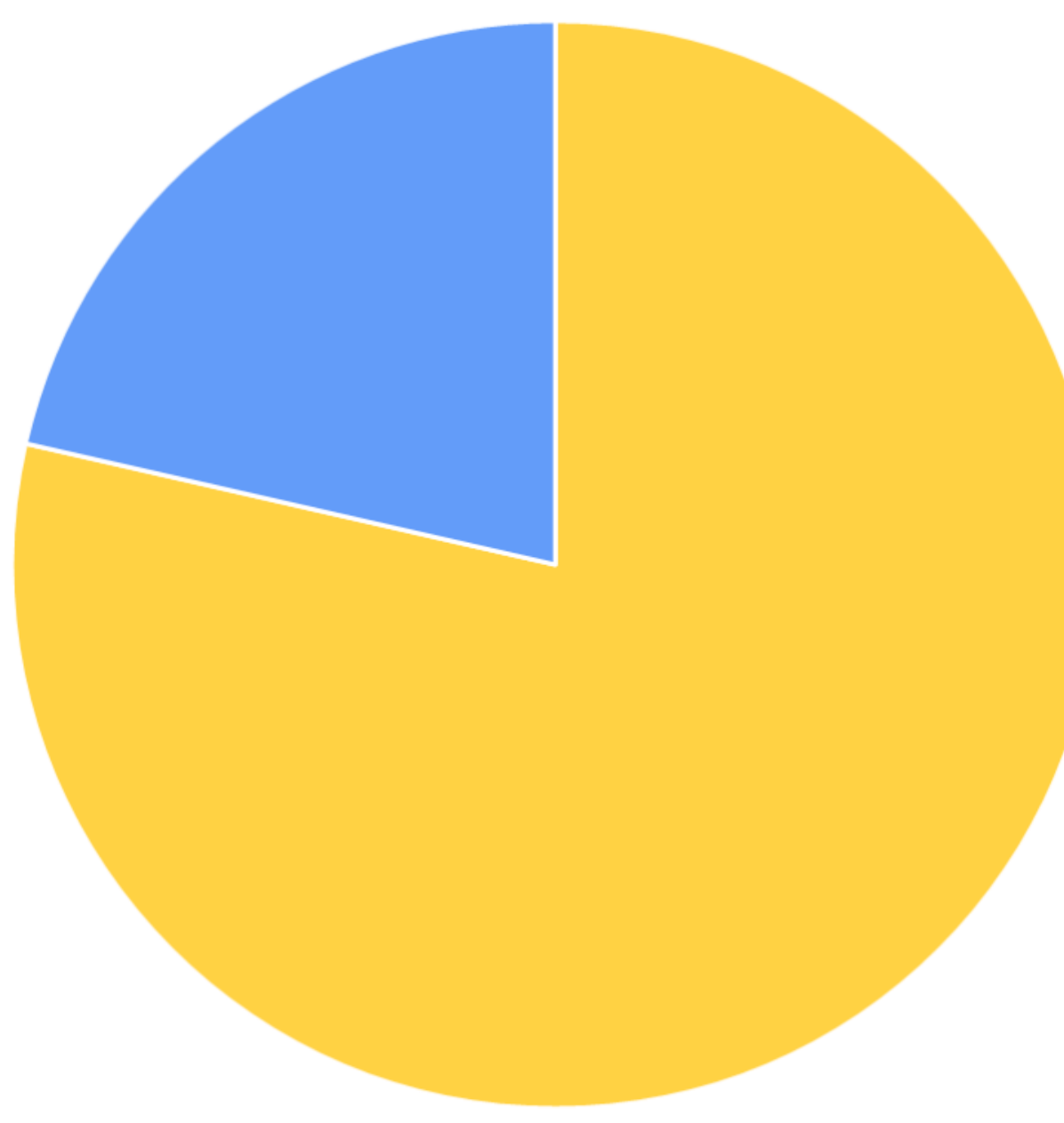

Fully documented - Not fully documented
Pre-implementation

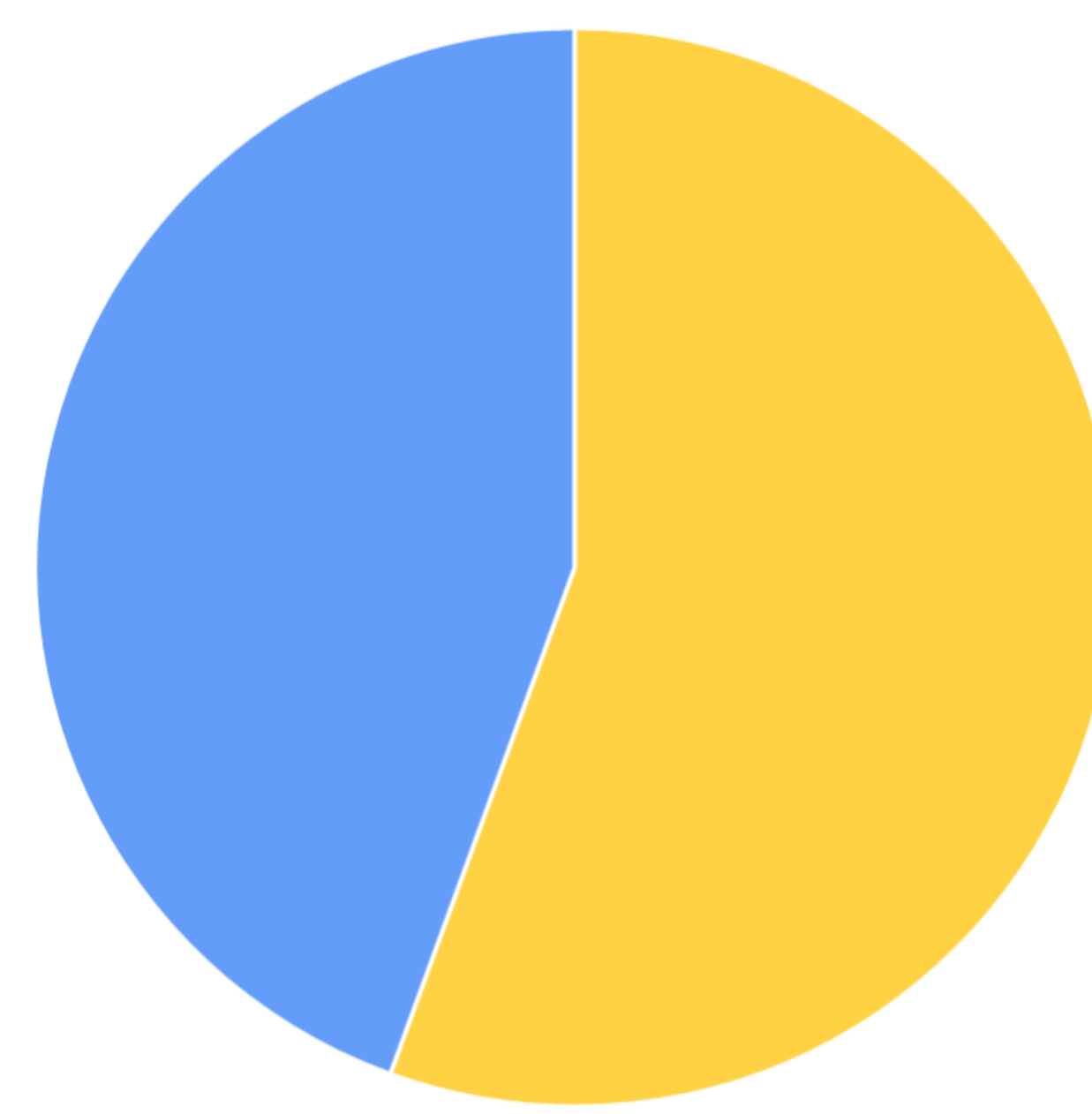

Fully documented

- Not fully documented

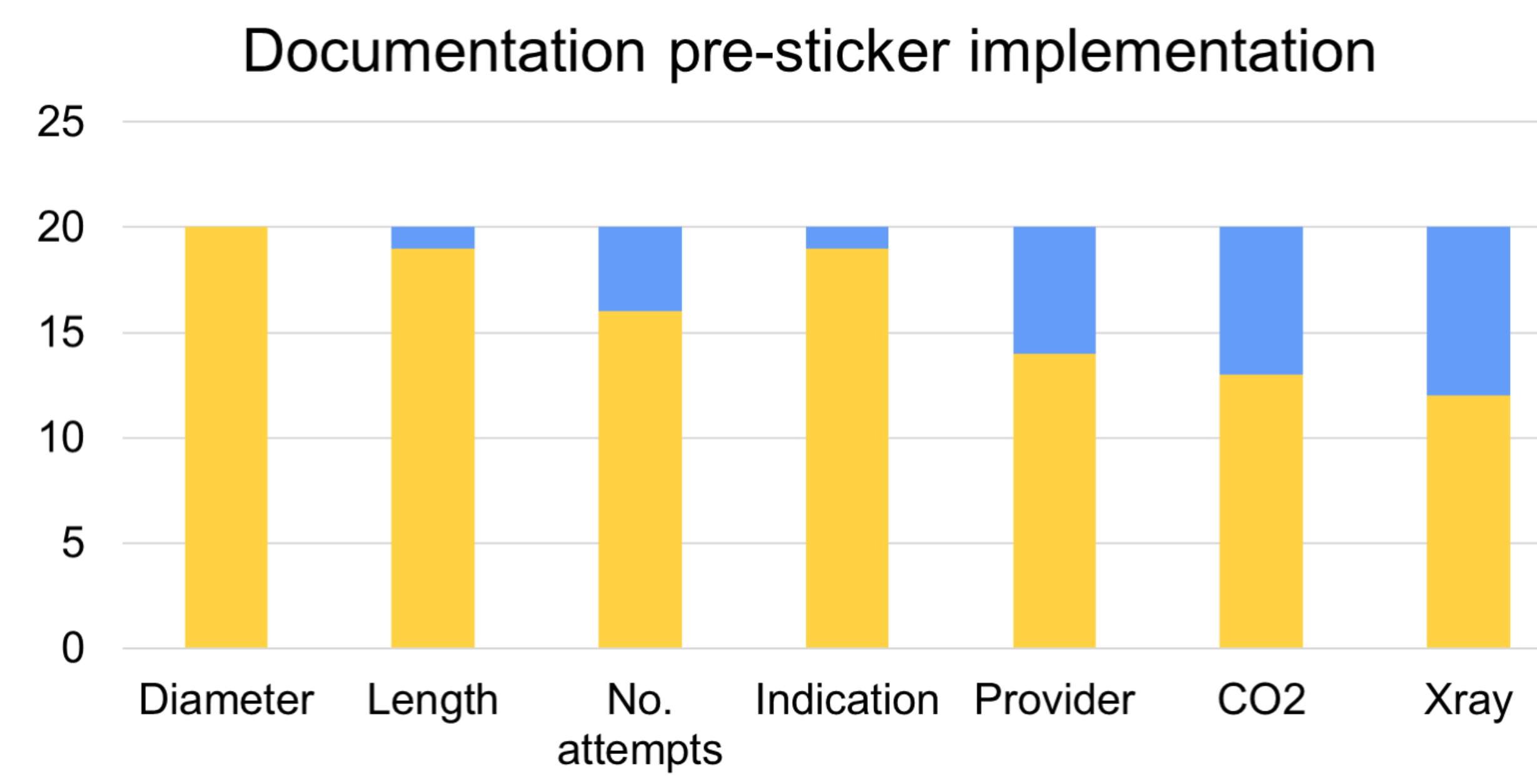

Documentation post-sticker implementation

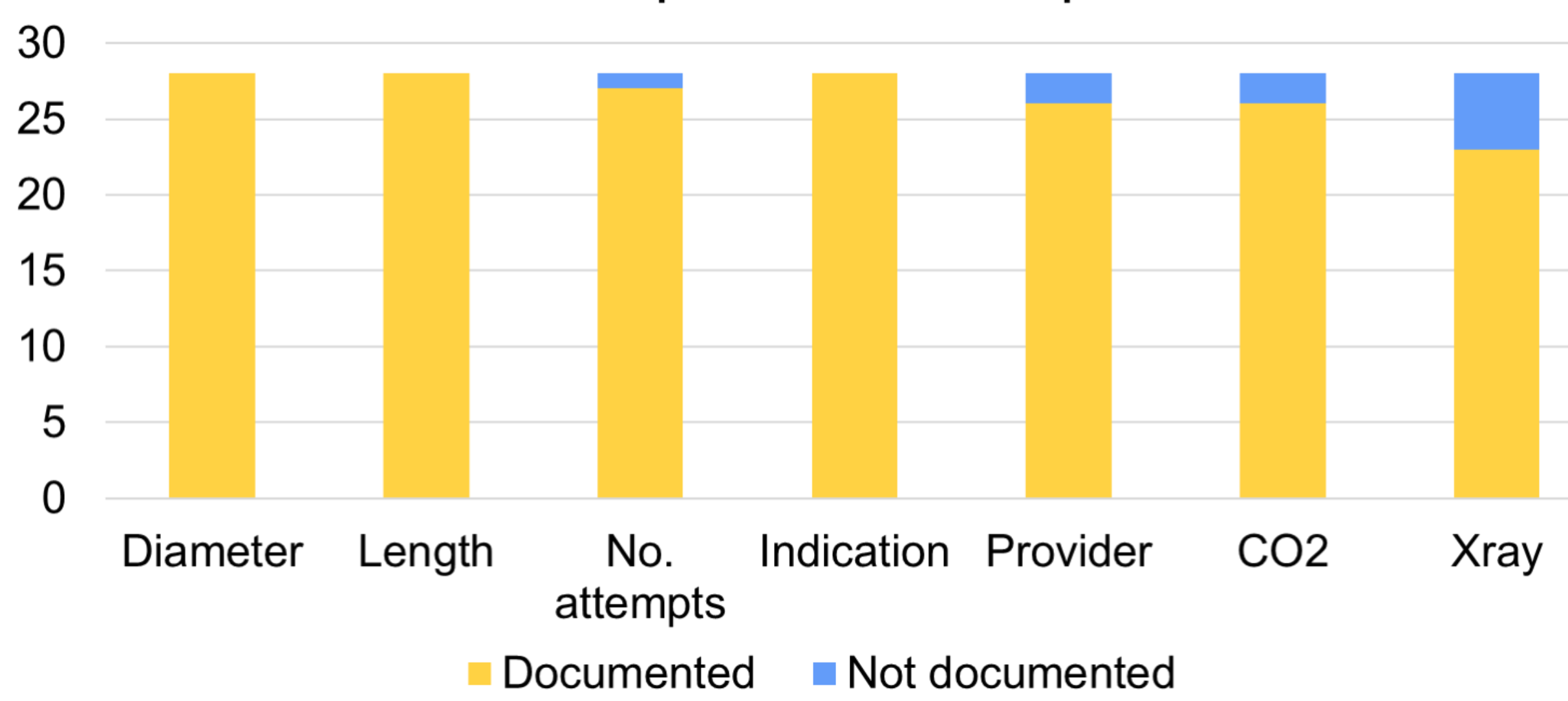

\section{Conclusions}

Introduction of a formal and structured checklist resulted in a significant improvement in documentation of endotracheal intubation attempts. Use of a checklist improves communication and patient safety during intubation. It also enables training opportunities within the team. We propose that a standardised checklist could be used in other units with likely improved training and patient outcomes. 